\title{
Patellar tendon buckling in post-operative total knee arthroplasty patients is more prominent than in healthy controls
}

\author{
Laura Chernak Slane $^{\mathrm{a}, *}$, Félix Dandois ${ }^{\mathrm{a}}$, Stijn Bogaerts ${ }^{\mathrm{a}, \mathrm{b}}$, Lennart Scheys ${ }^{\mathrm{a}, \mathrm{b}, 1}$, \\ Hilde Vandenneucker ${ }^{\mathrm{b}, 1}$ \\ ${ }^{a}$ KU Leuven, Institute for Orthopaedic Research and Training, UZ Pellenberg, Weligerveld 1/Blok 1, Pellenberg, Leuven 3212, Belgium \\ ${ }^{\mathrm{b}}$ University Hospitals Leuven, Campus Pellenberg, Pellenberg, Belgium
}

\section{A R T I C L E I N F O}

\section{Article history:}

Received 29 September 2018

Revised 30 March 2019

Accepted 7 June 2019

Available online $\mathrm{xxx}$

\section{Keywords:}

Knee flexion

Ultrasound

Biomechanics

Extensor mechanism

\begin{abstract}
A B S T R A C T
Recent evidence suggests the patellar tendon undergoes buckling during normal knee flexion, which likely contributes to the functioning of the extensor mechanism. Thus, evaluating buckling in patients following total knee arthroplasty (TKA), where extensor mechanism dysfunction remains a common complication, may be relevant. The study goals were to identify whether post-TKA patients exhibit differences in patellar tendon buckling from healthy, similarly-aged adults and whether such buckling correlates with knee and patellar tendon health. Patellar tendon buckling was assessed during passive knee flexion using ultrasound in post-TKA patients $(n=20 ; 12 \mathrm{M}, 68 \pm 8$ years $)$ and compared with previously reported data from healthy adults ( $n=12 ; 12 \mathrm{M} ; 70 \pm 8$ years). Patients exhibited significantly larger $(p<0.01)$ buckling magnitude and angles than healthy adults, and reduced distal buckling was linked with better Knee Society Scores $\left(p=0.04, R^{2}=0.24\right)$. The greater patellar tendon buckling observed in post-TKA patients could arise due to factors related to the surgery itself (e.g. infrapatellar fat pad resection) or it may be that post-TKA patients had greater patellar tendon buckling before their procedure. Alterations in patellar tendon buckling may predispose individuals to post-surgical complications including instability, anterior knee pain, and extensor mechanism dysfunction, with further work necessary to elucidate potential links.
\end{abstract} (C) 2019 IPEM. Published by Elsevier Ltd. All rights reserved.

\section{Introduction}

Total knee arthroplasty (TKA) is one of the most common orthopaedic procedures, with a prevalence of $4.55 \%$ among Americans over the age of 50 [1]. Although TKA success rates are relatively high, failure within 10 years still occurs in $2-8 \%$ of all procedures [2]. Common complications of TKA include instability [3-5], extension deficit, in which individuals are unable to actively reach full extension [4,5], and anterior knee pain [6]. The patellar tendon is a key contributor to knee function and may play a role contributing to these negative outcomes. Despite this, the complexity of patellar tendon function is still not fully understood. For example, recent evidence shows that the patellar tendon undergoes highly non-uniform motion [7-12] and is composed of fascicles with significantly heterogeneous mechanical properties [13-15]. In addition, recent evidence has suggested that the patellar tendon undergoes substantial buckling in which the tendon appears to wrinkle or fold back on itself when extended and passive

\footnotetext{
* Corresponding author.

E-mail address: laura.c.slane@gmail.com (L.C. Slane).

1 Both authors have equally contributed to this work.
}

[16], which may be relevant to knee joint function, though it remains poorly understood. Until recently, buckling of the patellar tendon during knee extension was suggested to be indicative of knee pathology [17-19]. Yet recent work has shown that patellar tendon buckling is largely present in healthy subjects [20], and is affected by age [21]. The clinical relevance of buckling has yet to be fully elucidated, but one hypothesis is that it may serve as a mechanical buffer protecting the tendon from sudden contractions, similar to the function of microstructural tendon crimp [22] and likely plays a role in the function of the extensor mechanism of the knee. Thus, the restoration of patellar tendon buckling during TKA may be relevant to the success of the procedure.

Therefore, the goals of this study were to (1) identify whether post-TKA patients exhibit patellar tendon buckling and at what flexion angles it occurs, (2) assess whether patellar tendon buckling is correlated with knee and patellar tendon health, and (3) evaluate whether patellar tendon buckling is different in post-TKA patients compared with healthy, similarly-aged, adults. In order to address our third goal, previously published data [21] collected by the same group and using the same methodology were included in the analysis. We hypothesized that post-TKA patients would exhibit patellar tendon buckling, with larger buckling magnitudes 
Table 1

Patient and healthy adult information. No differences between patients and healthy adults were found in terms of age, height, or maximum extension, but the healthy adults did have a significantly lower weight $(p=0.024)$, BMI $(p=0.008)$, and better VISA-P scores $(p=0.018)$. Knee Society Scores were not available for healthy adult subjects.

\begin{tabular}{lll}
\hline & Patients & Healthy adults $^{\mathrm{a}}$ \\
\hline Subjects & $n=20(12 \mathrm{M}, 8 \mathrm{~F})$ & $n=12(7 \mathrm{M}, 5 \mathrm{~F})$ \\
Age (years) & $68 \pm 8($ range: $56-77)$ & $70 \pm 8$ years (range: $56-80)$ \\
Height $(\mathrm{m})$ & $1.70 \pm 0.10$ & $1.69 \pm 0.12$ \\
Weight $(\mathrm{kg})$ & $84 \pm 14$ & $73 \pm 13$ \\
BMI & $29 \pm 5$ & $25 \pm 2$ \\
Max. extension & $8 \pm 6$ & $5 \pm 4$ \\
$\begin{array}{l}\text { (deg. of flexion) } \\
\text { VISA-P score }\end{array}$ & $69 \pm 19$ & $93 \pm 7$ \\
Knee Society Score & $88 \pm 8$ & - \\
\hline
\end{tabular}

a Health adult data were previously reported by Altinel et al. [20].

correlated with poor tendon health, and likewise larger buckling magnitudes compared with healthy adults.

\section{Methods}

Ethical approval for this study was received from the Commission for Medical Ethics UZ KU Leuven \#S59014. Patients recruited to this study had recently (10-16 months prior, mean: $14 \pm 2$ months) received a total knee arthroplasty completed by the same surgeon (author: H.V.). All patients received the same posterior-stabilized implant (Journey II, Smith \& Nephew, London, UK) with patellar resurfacing, and partial resection of the infrapatellar fat pad. All patients had been independently considered to have satisfactory procedures, defined as having no complications, and a satisfactory follow-up consultation. In total, 20 patients were recruited. Results were compared with previously reported data [21] from healthy, older adults (Table 1).

After providing written consent, all patients completed a VISA$P$ questionnaire to assess their current patellar tendon health [23]. Knee Society Scores [24] were also assessed by an orthopaeadic surgeon. Next, a twin-axis electric goniometer (Biometrics Ltd., Newport, UK) was secured to the skin, aligned with the midline of the femur at the iliotibial band and the midline of the fibula to enable knee angle measures. Patients were then seated in an isokinetic testing device (Biodex Medical Systems, Shirley, NY, USA) with their hip at approximately $90^{\circ}$. The maximum extension for each patient was determined by slowly moving the knee through extension to the point identified by patients as their maximum comfortable extension; this angle was then recorded. Cycles of passive flexion and extension between this peak extension and $90^{\circ}$ of flexion then commenced, with a six minute period used as a warm-up to precondition soft tissues [25].

Following the warm-up period, the machine was again used to induce flexion/extension cycles, with ultrasound radiofrequency (RF) data collected simultaneously. Data were collected from a $38 \mathrm{~mm}$ linear array transducer (L14-5/38, Ultrasonix Corporation, Richmond, BC, Canada) at $10 \mathrm{MHz}$ and 70 frames per second. The transducer was positioned randomly over either the proximal or distal tendon, with five trials of one cycle each collected from each transducer position. Knee angle and ultrasound data were synchronized using LabVIEW (National Instruments Corporation, Austin, TX, USA). As has been described previously [16], follow-up trials were performed with a hyperechoic wire positioned over the tendon to enable the measurement of tendon length changes during the cycle. To evaluate the magnitude of buckling, we measured the amount of tendon length that buckles, which is calculated as the difference in the distance between insertion points at maximum extension and maximum buckling (Fig. 1), normalized to the tendon length at $90^{\circ}$. Data were collected for three length trials from the proximal and distal transducer positions for all subjects [16].

Ultrasound post-processing was performed in MATLAB (R2015B, Mathworks, Natick, MA, USA). Images were imported and flexion/extension cycles were viewed frame-by-frame from extension to flexion. The ultrasound frame at which the tendon borders appeared continuous (e.g. without visible shadows from buckling) was identified, with the analysis repeated three times to reduce inaccuracy. The knee angle corresponding to this ultrasound frame was then termed the 'buckling angle' with the analysis repeated for all five distal and all five proximal trials. The shape of the buckle was also assessed with measurements made of the largest buckle in terms of its amplitude, width, and distance from the corresponding anatomical landmark (Fig. 1).

In addition to the ultrasound data, standard-of-care pre- and post-operative $\mathrm{x}$-rays of the knee were acquired for all subjects with the knee positioned at $30^{\circ}$ flexion. Scans were generally acquired one week preceding surgery, and at least six weeks postsurgery, though the scan dates did vary somewhat between patients (pre-operative range: $4 \mathrm{~m}-1 \mathrm{w}$, post-operative range: $6 \mathrm{w}-1 \mathrm{y}$ ). Next, from the flexion lateral view, a clinician measured the ratio of the patellar tendon length to the length of the patella, i.e. the Insall-Salvati index (ISI) [26].

All statistical analyses were performed in SPSS (SPSS Statistics v23, IBM, Armonk, NY) and Excel (2016 Analysis ToolPak, Microsoft Corporation, Redmond, WA), with $p<0.05$ taken as significant. First, the pre- and post-operative ISI were compared using paired $t$-tests. Next, to address our second research question, possible links between buckling results (distal buckling angle, proximal buckling angle and magnitude) and VISA-P and KSS scores were assessed using regression analyses in patients who exhibited buckling. To answer our third research question to see if there were any significant differences between buckling in post-TKA patients and healthy controls, $t$-tests were used to compare patients and healthy adults in terms of group characteristics (age, height, BMI, VISA-P score and Knee Society Score), and buckling results (distal buckling angle, proximal buckling angle, buckling magnitude, buckle amplitude, buckle width and buckle distance from anatomical landmark). Post-hoc, intra-class correlation (ICC) coefficients were computed to evaluate repeatability of distal and proximal buckling angle measures.

\section{Results}

Buckling was observed in 18 out of 20 patients, with 17 exhibiting both distal and proximal buckling. Distal buckling, which was observed in 18 subjects, occurred on average at $27 \pm 11^{\circ}$ of knee flexion. Proximal buckling was observed in 17 subjects and occurred at $24 \pm 10^{\circ}$ of flexion on average. No significant difference between pre- and post-operative Insall-Salvati Index was observed $(p=0.86)$.

Knee Society Scores of patients averaged $88 \pm 8$, which puts the average knee condition in the 'excellent' range (above 80 ), with even the low score of 70 qualifying as 'good' [27], which quantitatively confirms the clinical assessment that all included patients had satisfactory outcomes. No correlations between the VISA-P and buckling angles or magnitude were found. However, a significant correlation was found between Knee Society Score and distal buckling angle, with better Knee Society Scores correlated with smaller distal buckling angles $\left(p=0.04, R^{2}=0.24\right)$.

No differences between healthy adults and patients were found in terms of age, height, or maximum extension, but the healthy adults did have a significantly lower weight $(p=0.024)$, BMI $(p=0.008)$, and better VISA-P scores $(p=0.018)$. Both proximal and distal buckling angles of patients post-TKA were found to be significantly larger than what was previously observed in healthy older 


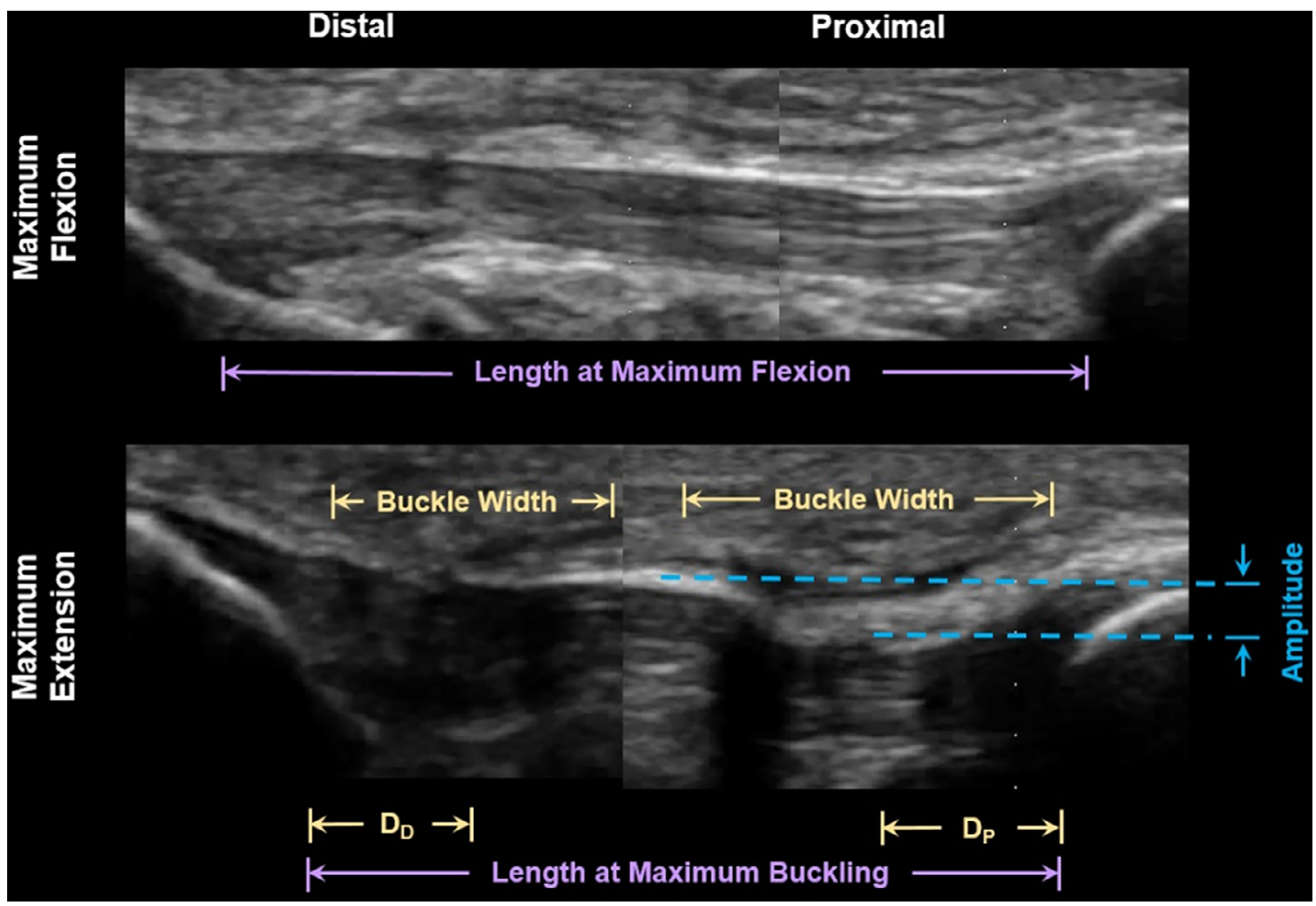

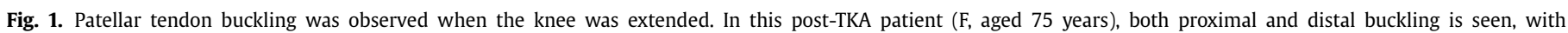

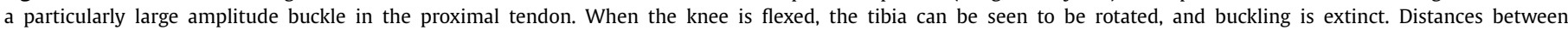

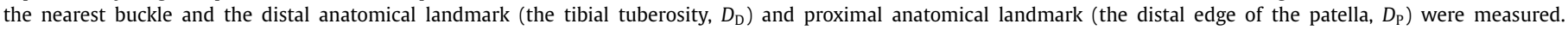

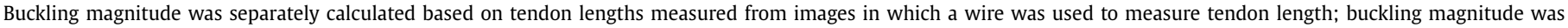
calculated as the difference in the distance between insertion points at maximum extension and maximum flexion.

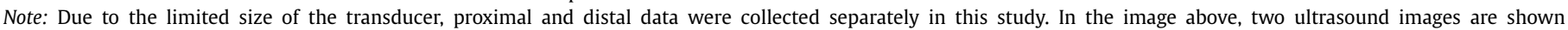
together for illustrative purposes.
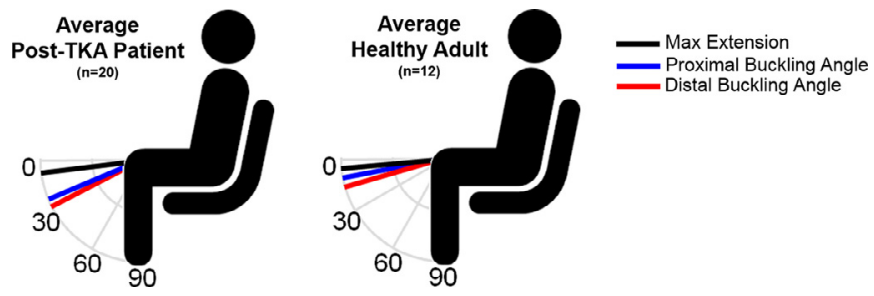

Fig. 2. Significant differences were observed between post-TKA patients and healthy adults in terms of proximal ( $24 \pm 10$ vs. $11 \pm 4^{\circ}$ of flexion, $\left.p<0.01\right)$ and distal ( $27 \pm 11$ vs. $15 \pm 8^{\circ}$ of flexion, $p<0.01$ ) buckling angles. No significant differences in maximum extension were observed ( $8 \pm 6$ vs. $5 \pm 4^{\circ}$ of flexion, $p=0.18$ ).

adults (Fig. 2), who exhibited a distal buckling angle of $15 \pm 8^{\circ}$ $(p=0.005)$ and proximal buckling angle of $11 \pm 4^{\circ}(p=0.006)$. Buckling magnitude was also significantly greater in TKA patients $(9.4 \pm 5.9 \%$ vs. $3.7 \pm 3.5 \% ; p=0.01)$. In addition, patients were observed to have a significantly greater amplitude in both distal (2.4 $\pm 1.2 \mathrm{~mm}$ vs. $1.4 \pm 0.7 \mathrm{~mm} ; p=0.009)$ and proximal $(2.3 \pm 1.4 \mathrm{~mm}$ vs. $1.0 \pm 0.6 \mathrm{~mm} ; p=0.04$ ) buckles, but no difference in terms of buckle width or distance from the anatomical landmark. ICC measures indicated good repeatability between repeat measures of distal and proximal buckling angles, with values of 0.98 and 0.97 , respectively, with $95 \%$ confidence interval above 0.93 .

\section{Discussion}

Patellar tendon buckling is a relatively understudied physiological phenomenon that may play an important role in the functioning of the knee joint. Thus, in TKA, where the aim is to restore normal knee function, it may be relevant to consider the buckling of the patellar tendon. The goal of this study was to assess whether current patients post-TKA are exhibiting similar patellar tendon buckling as healthy similarly-aged controls. In this study, we found that post-TKA patients did exhibit patellar tendon buckling, with better Knee Society Scores linked with smaller distal buckling angles. We also observed significant differences in patellar tendon buckling between post-TKA patients and healthy controls, with post-TKA patients exhibiting larger buckling angles, greater buckling magnitudes and greater amplitude buckles.

In contrast with our hypothesis, we found that patients exhibited significantly larger buckling angles, and buckling magnitudes compared with healthy, similarly-aged adults. These results were unexpected, as the patients recruited for this study were assessed and considered to have satisfactory outcomes following their procedure. However, these results are consistent with prior studies that linked extreme patellar tendon buckling with pathology [17-19]. Likewise, we also observed that among patients, better performance on the Knee Society Score was correlated with smaller distal buckling angles. Although the clinical role of patellar tendon buckling has yet to be elucidated, it appears to be an almost universal phenomenon inherent in knee flexion. One hypothesis is that such buckling may serve to protect the tendon from high strains or sudden contractions in a way similar to how microstructural tendon crimp is hypothesized to serve as a protective mechanism enabling the tendon to achieve high strains without injury [22]. Likewise, changes in buckling post-TKA could be an understudied contributor to poor long-term outcomes. For example, a sudden increase in buckling in the tendon could lead to reduced joint stability, and/or reduced ability of patients to reach full extension (i.e. extension deficit), two known complications of 
TKA procedures $[4,5,28]$. Significantly reduced buckling, although not observed here, could also be problematic; a sudden reduction in buckling could expose the tendon to greater strains, increase the risk of chronic tendon injury, and potentially lead to anterior knee pain, a known complication of total knee arthroplasty [29]. Thus, patellar tendon buckling may be a relevant factor to consider, along with other common clinical parameters (e.g. collateral ligament balancing), during TKA procedures.

Unfortunately, in this study, without pre-operative buckling data, it is unclear whether differences in patellar tendon buckling occur because of the TKA, or if the increased buckling in some way contributed to the need for a TKA. In the case of the first, this would suggest that something in the surgical procedure itself, or in the recovery process, affects patellar tendon buckling. In terms of the surgical procedure, patella positioning, infrapatellar fat pad resection, changes in patellar tracking post-TKA, or even microtrauma from the surgery itself could influence patellar tendon buckling. Comparisons of ISI pre- and post-TKA showed no significant changes in the ISI, suggesting that patella positioning was not significantly altered during the surgical procedure. Infrapatellar fat pad resection may also have played a role in altering patellar tendon buckling. Although a common clinical procedure [30], there is increasing evidence that such resections may influence the patellar tendon, both in terms of anterior knee pain and patellar tendon length, though evidence is conflicting [30]. Interestingly, in addition to the significantly higher buckling magnitude in patients, we also noted that patients seemed to exhibit a more extreme form of buckling, with significantly higher amplitude buckles, compared with healthy adults (Fig. 1). The removal of fat pad tissue might account for this difference, as without the underlying tissue, there is greater joint space for buckling. Future studies testing this hypothesis could theoretically quantify the amount of fat pad resection, and/or use a subject-specific finite element model, to test how fat pad resection may be linked with changes in patellar tendon buckling.

On the other hand, it is possible that patients who received TKAs may have already had differences in patellar tendon buckling relative to healthy adults, which could even have contributed to their pathology. For example, knee osteoarthritis has been shown to contribute to quadriceps weakness [31]. Weaker quadriceps would have presumably less passive stiffness [32] which, due to the patellar tendon's anatomical function as part of the quadriceps muscle-tendon unit [33,34], would most likely increase buckling within the patellar tendon. A potential effect of such extreme buckling is joint instability, which can lead to abnormal knee joint loading and increased degenerative wear [35]. Pre-operative differences in patellar tracking could also be relevant to consider. In future studies, it would certainly be of interest to study patients prior to surgery, though pain could be a limiting factor during data collection. Alternatively, patients with similar radiographic damage but significant differences in pain or function could be evaluated to assess whether there are any links between function, pain and patellar tendon buckling.

An interesting observation is that this study suggested that smaller bucking angles may be better than larger buckling angles, with both healthy adults and better functioning patients (as measured with KSS) exhibiting smaller buckling angles. In contrast, our prior work on age-related changes [21] showed greater buckling angles in young adults $\left(23 \pm 8^{\circ}\right)$ compared with middle-aged (15 $\left.\pm 4^{\circ}\right)$ and older adults $\left(15 \pm 8^{\circ}\right)[16,21]$. These results together suggest a new hypothesis regarding patellar tendon buckling that the link between buckling and knee health may be complex and it may be that both too little and too much buckling is maladaptive. Whereas too little buckling may expose the joint to high strains, too much buckling could lead to joint instability. This hypothesis further suggests that there may be an optimal functional range of buckling, as there are optimal ranges for many other musculoskeletal parameters (e.g. muscle force-length relationship [18,19]) and understanding how this functional range may vary within different subject groups could deepen our understanding of the role that buckling plays in soft tissue mechanics. Recent observations of buckling in other tissues (e.g. the Achilles [36]) may suggest that buckling is a common aspect of soft tissue mechanics, but more work is clearly needed to better elucidate such findings. Also in this study, patellar tendon buckling was observed in all but two patients, which is consistent with a growing body of work suggesting that buckling is broadly present and likely an inherent characteristic of knee function $[16,21]$. It is unclear why two of the patients did not exhibit buckling, though most likely this was related to an inability to fully relax in the experimental setup. We have previously observed that buckling occurs in some individuals only in a hyperextended position [16], and older adults with a TKA may have been hesitant to reach such an extended posture. Other work on middle-aged and older adults suggests that inadvertent contraction of the quadriceps may make it difficult to observe buckling experimentally [21], which most likely accounts for the lack of observed buckling in two patients. More work is certainly needed to better clarify the function and prevalence of patellar tendon buckling in order to understand its clinical role.

There are some limitations to this study. The first is the overall sample size is small $(n=32)$, and the convenience sample of patients and controls means there may have been selection bias. A second limitation is that without pre-operative buckling data, it is unclear whether buckling was altered specifically due to the procedure, or whether patients who need a TKA may have altered patellar tendon buckling pre-operatively. By adding a longitudinal assessment of buckling in patients before and after surgery, and also by quantifying surgical factors such as infrapatellar fat pad resection and patellar tracking, the influence of the surgical procedure itself on patellar tendon buckling may become more clear. A future study with a longitudinal component could also include a longerterm follow-up of patients; in this study, patients were assessed on average 10 months post-surgery which may be an insufficient amount of time for assessing long-term changes [30]. An inherent limitation of this study is the use of a 2D imaging modality (ultrasound) to characterize the shape of 3D tissue. Imaging in 3D could better characterize how buckling alters tendon shape as well as evaluate whether buckling varies mediolaterally. Additionally, future work could aim to assess whether there are fundamental differences in patellar tendon buckling in patients with unsatisfactory outcomes.

\section{Conclusions}

There is increasing evidence that patellar tendon buckling is an inherent component of knee joint behavior. Post-TKA patients were observed to exhibit significantly greater patellar tendon buckling angles, with greater buckling magnitudes compared with healthy, similarly-aged, adults. Surgical factors, such as infrapatellar fat pad resection may contribute to the increased patellar tendon buckling observed. However, this could also arise due to post-surgical factors, or even contribute to the need for treatment in the first place. Alterations in patellar tendon buckling may predispose individuals to post-surgical complications including instability, anterior knee pain, and extensor mechanism dysfunction. Further work is warranted to better understand the links between patellar tendon buckling, TKA and post-surgical outcomes, to assess whether buckling should be considered intraoperatively.

\section{Conflict of interest}

None. 


\section{Funding}

This work was supported by the European Knee Society and the National Institutes of Health [F32 AR069459-01].

\section{Ethical approval}

Ethical approval for this study was received from the Commission for Medical Ethics UZ KU Leuven \#S59014.

\section{Acknowledgments}

We thank Lore Hermans and Kristel Van de Loock for their assistance with subject recruitment, and Hans Geelen for his assistance computing ISI values

\section{References}

[1] Maradit Kremers H, Larson DR, Crowson CS, Kremers WK, Washington RE, Steiner CA, et al. Prevalence of total hip and knee replacement in the United States. J Bone Joint Surg Am 2015;97:1386-97. doi:10.2106/JBJS.N.01141.

[2] McCalden RW, Robert CE, Howard JL, Naudie DD, McAuley JP, MacDonald J S. Comparison of outcomes and survivorship between patients of different age groups following TKA. J Arthroplasty 2013;28:83-6.

[3] Fehring TK, Odum S, Griffin WL, Mason JB, Nadaud M. Early failures in total knee arthroplasty. Clin Orthop Relat Res 2001:315-8.

[4] Sharkey PF, Hozack WJ, Rothman RH, Shastri S, Jacoby SM. Why are total knee arthroplasties failing today? Clin Orthop Relat Res 2002:7-13.

[5] Sharkey PF, Lichstein PM, Shen C, Tokarski AT, Parvizi J. Why are total knee arthroplasties failing today-has anything changed after 10 years? J Arthroplasty 2014;29:1774-8. doi:10.1016/j.arth.2013.07.024.

[6] Petersen W, Rembitzki IV, Brüggemann G-P, Ellermann A, Best R, Koppenburg AG, et al. Anterior knee pain after total knee arthroplasty: a narrative review. Int Orthop 2014;38:319-28. doi:10.1007/s00264-013-2081-4.

[7] Lee D, Barrett R, Ryan M, Saxby DJ, Newsham-West R, Obst SJ. In vivo strain in the deep and superficial regions of the human patellar tendon. Scand J Med Sci Sports 2016. doi:10.1111/sms.12721.

[8] Pearson SJ, Mohammed ASA, Hussain SR. Patellar tendon in vivo regional strain with varying knee angle. J Biomech 2017. doi:10.1016/j.jbiomech.2017.06.038.

[9] Pearson SJ, Ritchings T, Mohamed ASA. Regional strain variations in the human patellar tendon. Med Sci Sports Exerc 2014;46:1343-51. doi:10.1249/MSS. 0000000000000247.

[10] Slane L, Bogaerts S, Thelen DG, Scheys L. Non-uniform deformation of the patellar tendon during passive knee flexion. J Appl Biomech Press 2017;34:14-22.

[11] Almekinders LC, Vellema JH, Weinhold PS. Strain patterns in the patellar tendon and the implications for patellar tendinopathy. Knee Surg Sports Traumatol Arthrosc 2002;10:2-5. doi:10.1007/s001670100224.

[12] Gejo R, Morita Y, Matsushita I, Sugimori K, Watanabe H, Kimura T. Intraoperative patellar tendon strain: predicting the range of knee flexion after total knee arthroplasty. J Orthop Sci 2009;14:51-5. doi:10.1007/s00776-008-1286-7.

[13] Basso O, Amis AA, Race A, Johnson DP. Patellar tendon fiber strains: their differential responses to quadriceps tension. Clin Orthop Relat Res 2002:246-53.

[14] Basso O, Johnson DP, Amis AA. The anatomy of the patellar tendon. Knee Surg Sport Traumatol Arthrosc 2001;9:2-5.

[15] Hansen P, Haraldsson BT, Aagaard P, Kovanen V, Avery NC, Qvortrup K, et al. Lower strength of the human posterior patellar tendon seems unrelated to mature collagen cross-linking and fibril morphology. J Appl Physiol 2010;108:4752. doi:10.1152/japplphysiol.00944.2009.

[16] Slane LC, Bogaerts S, Mihejeva I, Scheys L. Evidence of patellar tendon buckling during passive knee extension. Knee 2016;23. doi:10.1016/j.knee.2016.06.005.
[17] Schweitzer ME, Mitchell DG, Ehrlich SM. The patellar tendon: thickening, internal signal buckling, and other MR variants. Skeletal Radiol 1993;22:411-16.

[18] Berlin R, Levinsohn EM, Chrisman H. The wrinkled patellar tendon: an indication of abnormality in the extensor mechanism of the knee. Skeletal Radiol 1991;20. doi:10.1007/BF00241662.

[19] Reiff D, Heenan S, Heron C. MRI appearances of the asymptomatic patellar tendon on gradient echo imaging. Skeletal Radiol 1995;24. doi:10.1007/ BF00198074.

[20] Altinel L, Kose KC, Degirmenci B, Petik B, Acarturk G, Colbay M. The midterm effects of diabetes mellitus on quadriceps and patellar tendons in patients with knee arthrosis: a comparative radiological study. J Diabetes Complications 21:392-6. doi:10.1016/j.jdiacomp.2006.07.003.

[21] Slane LC, Dandois F, Bogaerts S, Vandenneucker H, Scheys L. Patellar Tendon buckling is altered with age. Med Eng Phys 2018:59:15-20. https://www. sciencedirect.com/science/article/abs/pii/S1350453318301139.

[22] Patterson-Kane J, Firth E, Goodship A, Parry D. Age-related differences in collagen crimp patterns in the superficial digital flexor tendon core region of untrained horses. Aust Vet J 1997;75:39-44. doi:10.1111/j.1751-0813.1997. tb13829.x.

[23] Visentini PJ, Khan KM, Cook JL, Kiss ZS, Harcourt PR, Wark JD. The VISA score: an index of severity of symptoms in patients with jumper's knee (patellar tendinosis). Victorian Institute of Sport Tendon Study Group. J Sci Med Sport $1998 ; 1: 22-8$.

[24] Kurer M, Gooding C. Knee Society Score. Orthop Scores n.d. http://www. orthopaedicscore.com/scorepages/knee_society_score.html (accessed 19 June 2017).

[25] Hawkins D, Lum C, Gaydos D, Dunning R. Dynamic creep and pre-conditioning of the Achilles tendon in-vivo. J Biomech 2009;42:2813-17. doi:10.1016/j. jbiomech.2009.08.023.

[26] Insall J, Salvati E. Patella position in the normal knee joint. Radiology 1971;101:101-4. doi:10.1148/101.1.101.

[27] Asif S, Choon D. Midterm results of cemented press fit condylar sigma total knee arthroplasty system. J Orthop Surg 2005;13:280-4. doi:10.1177/ 230949900501300311.

[28] Wilson CJ, Theodoulou A, Damarell RA, Krishnan J. Knee instability as the primary cause of failure following Total Knee Arthroplasty (TKA): a systematic review on the patient, surgical and implant characteristics of revised TKA patients. Knee 2017;24:1271-81. doi:10.1016/j.knee.2017.08.060.

[29] Breugem SJM, Haverkamp D. Anterior knee pain after a total knee arthroplasty: What can cause this pain? World J Orthop 2014;5:163-70. doi:10.5312/wjo.v5. i3.163.

[30] Van Beeck A, Clockaerts S, Somville J, Van Heeswijk JHW, Van Glabbeek F, Bos PK, et al. Does infrapatellar fat pad resection in total knee arthroplasty impair clinical outcome? A systematic review. Knee 2013;20:226-31. doi:10. 1016/j.knee.2013.01.005.

[31] Johnson GA, Tramaglini DM, Levine RE, Ohno K, Choi N-Y, Woo SL-Y. Tensile and viscoelastic properties of human patellar tendon. J Orthop Res 1994;12:796-803. doi:10.1002/jor:1100120607.

[32] Leite DX, Vieira JMM, Carvalhais VOC, Araújo VL, Silva PLP, Fonseca ST. Relationship between joint passive stiffness and hip lateral rotator concentric torque. Rev Bras Fisioter 2012;16:414-21. doi:10.1590/S1413-35552012005000046.

[33] Toumi H, Higashiyama I, Suzuki D, Kumai T, Bydder G, McGonagle D, et al. Regional variations in human patellar trabecular architecture and the structure of the proximal patellar tendon enthesis. J Anat 2006;208:47-57. doi:10.1111 j.1469-7580.2006.00501.x.

[34] Rumian AP, Wallace AL, Birch HL. Tendons and ligaments are anatomically distinct but overlap in molecular and morphological features - a comparative study in an ovine model. J Orthop Res 2007;25:458-64. doi:10.1002/jor.20218.

[35] Reinders J, Sonntag R, Kretzer JP. Wear behavior of an unstable knee: a stabilization via implant design? Biomed Res Int 2014;2014. doi:10.1155/2014/ 821475.

[36] Kinugasa R, Taniguchi K, Yamamura N, Fujimiya M, Katayose M, Takagi S, et al A multi-modality approach towards elucidation of the mechanism for human achilles tendon bending during passive ankle rotation. Sci Rep 2018. doi:10. 1038/s41598-018-22661-7. 